\title{
Left adrenal gland metastasis of breast invasive ductal carcinoma: A case report
}

\author{
TAO HE ${ }^{1,2^{*}}$, JIAJU LIU ${ }^{1,3^{*}}$, YIFAN LI ${ }^{1,4}$, LU JIN $^{1,4}$, SHUOLEI SUN ${ }^{1}$, LIANGCHAO NI $^{1}$, \\ XIANGMING MAO ${ }^{1}$, SHANGQI YANG ${ }^{1}$ and YONGQING LAI ${ }^{1}$ \\ ${ }^{1}$ Department of Urology, Peking University Shenzhen Hospital, Shenzhen 518036; \\ ${ }^{2}$ Guangzhou Medical University, Guangzhou 511436; ${ }^{3}$ Shantou University Medical College, \\ Guangdong 515041; ${ }^{4}$ Anhui Medical University, Anhui, Hefei 230032, P.R. China
}

Received September 3, 2015; Accepted February 25, 2016

DOI: $10.3892 / \mathrm{mco} .2016 .814$

\begin{abstract}
The majority of the metastatic lesions of the adrenal gland normally originate from lung cancer, colon malignant tumor, renal cell carcinoma and melanoma. However, adrenal gland metastasis that metastasize from breast invasive ductal carcinoma are extremely rare. The present study reported a rare case of left adrenal gland metastasis in a 35-year-old female who was diagnosed as breast carcinoma 5 years ago with a mass located on the left adrenal gland, which was detected during a routine examination. The patient was asymptomatic and adrenal gland computed tomography revealed a mass in the left adrenal gland. Definitive preoperative diagnosis failed to be established. Left adrenal gland laparoscopic adrenalectomy was performed and the diagnosis of adrenal gland metastasis of breast invasive ductal carcinoma was confirmed by pathological and immunohistochemical examination. The patient remained in good condition by the time of writing.
\end{abstract}

\section{Introduction}

The majority of the common primary neoplasms which metastasize to the adrenal gland include melanoma, lung cancer, breast carcinoma, colon malignant tumor and renal cell carcinoma (1). In practice, adrenal gland metastasis normally results from systemic metastasis, particularly in the advanced stage of the primary neoplasms like breast carcinoma, and the most common method of metastasis is blood-borne $(2,3)$. Breast carcinoma is the most common malignancy in females,

Correspondence to: Professor Yongqing Lai or Professor Shangqi Yang, Department of Urology, Peking University Shenzhen Hospital, 1120 Lianhua Road, Shenzhen 518036, P.R. China

E-mail: yqlord@163.com

E-mail: yangshangqi88@aliyun.com

*Contributed equally

Key words: adrenal gland metastasis, breast invasive ductal carcinoma, laparoscopic adrenalectomy while the primary histological type of the breast cancer is invasive ductal carcinoma. A report revealed that $\sim 80 \%$ of all breast cancer types are breast invasive ductal carcinomas (4), which normally metastasize to the lung, liver, bone and brain; however, metastasis to the adrenal gland is rare (5). Metastasis of breast carcinoma often leads to a poorer prognosis and a lower 5-year survival rate (6). Patients who have solitary adrenal gland metastasis normally have a long-term survival compared with those who exhibit synchronous metastasis (7). Laparoscopic adrenalectomy (LA), the treatment of adrenal gland metastases, has become more and more acceptable.

The present study reported a rare case of left adrenal gland metastasis of breast invasive ductal carcinoma, in good condition, following treatment with LA. This case is the newest and the sixth to be presented in the literature. The present study was approved by the Ethics Committee of Peking University Shenzhen Hospital (Shenzhen, China) and the written informed consent was provided by the patient.

\section{Case report}

A 35-year-old female, who was found to have a mass located on the left adrenal gland during a routine examination by positron emission tomography/computed tomography (PET/CT) in Shenzhen Armed Police Hospital (Shenzhen, China) on May $15^{\text {th }} 2015$. The PET/CT revealed a nodular hypermetabolic focus located on the left adrenal gland, measuring $2.5 \times 2.6 \times 2.8 \mathrm{~cm}$, and the maximum standardized uptake value was 3.9 (Fig. 1). Therefore, the possibility of metastasis was considered. The patient was subsequently admitted to the Department of Urology, Peaking University Shenzhen Hospital (Shenzhen, China) for further examination and therapy on June $1^{\text {st }} 2015$. The patient was diagnosed with left breast cancer 5 years ago and had undergone mastectomy, followed by regular chemotherapy and radiotherapy. The patient was asymptomatic with a normal appetite, no abdominal pain and no weight changes. Additionally, urinary, respiratory, cardiovascular or constitutional symptoms were negative. Physical examination revealed a well-developed and well-nourished female whose pulse, temperature, blood pressure and respiration rate were all within normal limits. The chest was clear to percussion and auscultation, and no masses were palpable on abdominal examination. Laboratory 
examinations, including routine hematological examination and biochemical tests, were within normal limits. The plasma adrenocorticotropic hormone and cortisol levels were within a normal range. An adrenal CT scan, enhanced adrenal CT and three-dimensional imaging were performed, which revealed a $2.1 \times 2.3 \times 2.4 \mathrm{~cm}$ round abnormal density shadow [23-26 Hounsfield units (HU)] in the lateral branches of the left adrenal gland with clear and smooth edges. Also, the density of the shadow was heterogeneous (Fig. 2). Enhanced CT revealed a mild to moderate strengthening of the mass (33-63 HU; Fig. 2).

The left adrenal gland LA was performed on June $9^{\text {th }} 2015$. A tumour with clear margin, $2.5 \mathrm{~cm}$ in diameter, was located on the upside of the left kidney and the tumour exhibited mild adhesion with the surrounding tissue. Normal adrenal gland tissue was not observed. The pathological report revealed irregular nest clusters, adenous duct-like heterotypic cells in the adrenal gland tissue, the growth of the cells were infiltrative and piecemeal necrosis was observed (Fig. 3). The result of immunohistochemistry was as follows: Estrogen receptor (+), progesterone receptor $(+)$ and human epidermal growth factor-2 (+/++; Fig. 3). The diagnosis of adrenal gland metastasis of breast invasive ductal carcinoma was subsequently confirmed.

The patient was discharged on the 7 days post-operation. At the time of writing, no problems were reported with the patient.

\section{Discussion}

Adrenal gland metastasis, metastasized from breast invasive ductal carcinoma, are extremely rare. Only 5 cases, to the best of our knowledge, have been reported previously in the literature (Table I) $(2,5,6,8,9)$. The present case is the newest and the sixth in the literature. A previous study by Borst and Ingold (10), including 2,246 patients with breast invasive ductal carcinoma, revealed no adrenal gland metastasis in those patients.

The majority of patients with adrenal gland metastases are asymptomatic, while a small proportion of the patients may present adrenal gland disfunction if the malignant mass replaces or destroys the normal adrenal gland tissues (6). Although primary adrenal gland malignancies like adrenocortical carcinomas usually secrete hormone excessively and cause certain relative symptoms, or are followed with other symptoms, including hemorrhage, abdominal distension or discomfort (11), metastatic adrenal gland carcinomas are often difficult to identify from primary adrenal gland malignancies, which can lead to misdiagnosis (5). Each patient who has a history of malignance and mass $>2 \mathrm{~cm}$ in diameter in adrenal gland must be highly considered as a possible case of adrenal gland metastasis (1). Normally, a CT scan and magnetic resonance imaging can be used as a prior option to identify primary carcinoma and metastases of the adrenal gland (5). The patient in the present case was asymptomatic with no abdominal pain or other problems; however, she had a history of left breast carcinoma and a PET/CT revealed a nodular hypermetabolic focus located on the left adrenal region, measuring $2.5 \times 2.6 \times 2.8 \mathrm{~cm}$, which was considered as a possible metastasis.

LA has become the acceptable and is currently the first option of surgical treatment in adrenal gland tumors (1) as it has

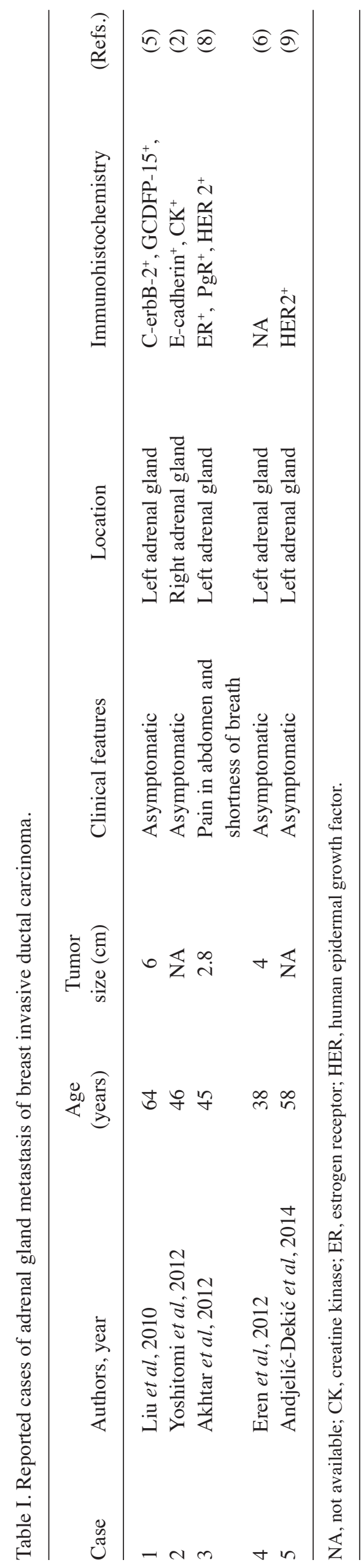




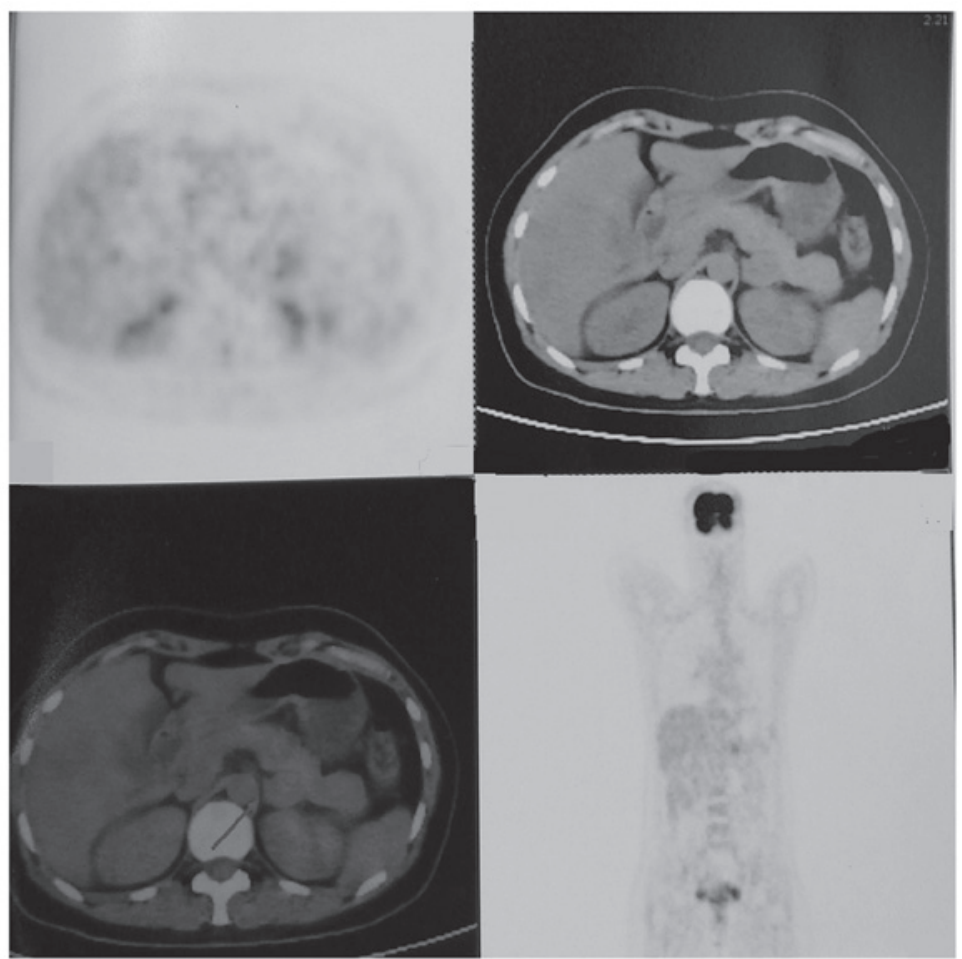

Figure 1. Results of adrenal gland positron emission tomography/computed tomography. The images revealed a nodular hypermetabolic focus located on the left adrenal gland region, measuring $2.5 \times 2.6 \times 2.8 \mathrm{~cm}$, and the maximum stadardized uptake value was 3.9.
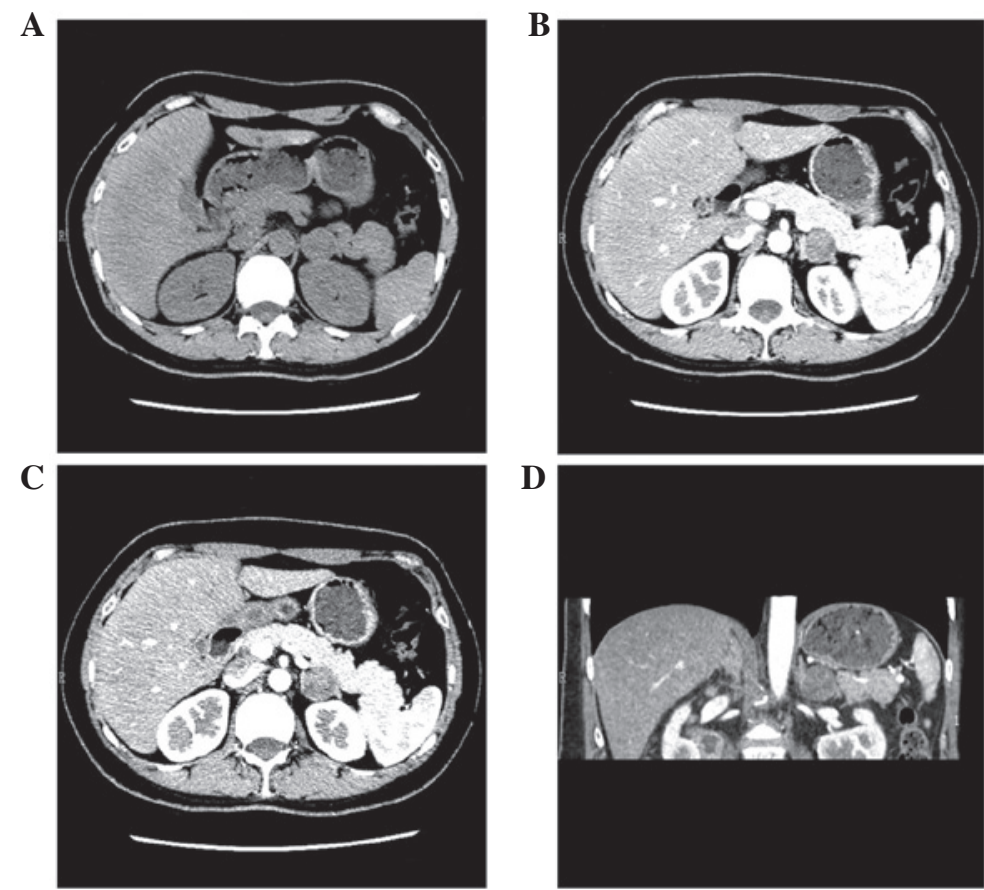

Figure 2. Results of adrenal gland CT scan and enhanced adrenal gland CT. (A) An adrenal gland CT scan revealed a $2.1 \times 2.3 \times 2.4 \mathrm{~cm}$ round abnormal density shadow (23-26 HU) in the lateral branches of the left adrenal gland with clear and smooth edges. The density of the shadow was heterogeneous. (B-D) The enhanced adrenal gland CT revealed a mild to moderate strengthening of the mass (33-63 HU). CT, computed tomography; HU, Hounsfield units.

many advantages, including reduced requirement of analgesia following surgery and the patient being discharged earlier (11). However, while performing LA to resect primary carcinoma or metastasis of the adrenal gland, carcinomatosis and port-site metastasis, which may be caused by several factors, including the behavior of the tumor, patient's condition and the operation of surgery, should be taken into consideration as they have been reported previously (12). It is also important to avoid port-site metastasis and carcinomatosis when performing LA. Therefore, whether LA can be widely applied to adrenal gland tumors remains controversial (13). If it is necessary to perform adrenalectomy, particularly for bilateral adrenal 

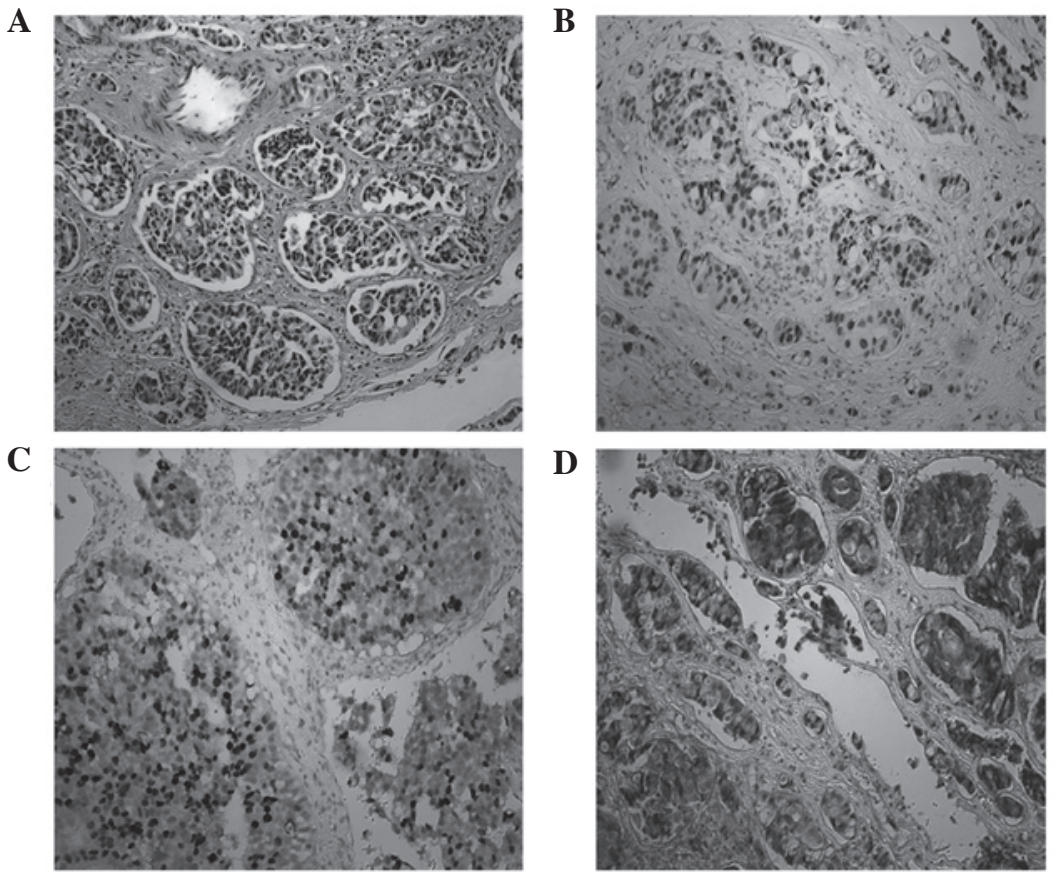

Figure 3. Results of pathological and immunohistochemical examination. (A) The pathological examination revealed that the adrenal gland tumor tissue was composed of irregular nest clusters, adenous duct-like heterotypic cells and the growth of the cells were infiltrative. Piecemeal necrosis was also observed (magnification, x200). Immunohistochemical staining (magnification, x200) of the tumor cells demonstrated positive staining for (B) estrogen receptor (+), (C) progesterone receptor (+) and (D) human epidermal growth factor-2 (+/++).

gland metastasis, partial adrenalectomy is an alternative and ideal treatment strategy. When performing partial adrenalectomy, minimally invasive surgical procedures, including laparoscopic or robotic procedures, are ideal options, which may assist in avoiding endocrinological complications caused by adrenalectomy, including iatrogenic Addison's disease (14). Notably, combining this with radiotherapy, chemotherapy and hormone replacement therapy can provide an improved outcome in prolonging survival (5).

In conclusion, a case of adrenal gland metastasis of breast invasive ductal carcinoma was reported in the present study. The metastasis of breast carcinoma to the adrenal gland is rare, therefore it is important to identify metastasis and primary adrenal gland tumors. Definite diagnosis can be established by $\mathrm{CT}$ and pathological examination. As one of treatments of adrenal gland metastasis, the role of LA in prolonging survival remains to be further elucidated in future studies.

\section{Acknowledgements}

The present study was supported by the National Natural Science Foundation of China (no. 81101922), the Science and Technology Development Fund Project of Shenzhen (nos. JCYJ20130402114702124 and JCYJ20150403091443329) and the fund of Guangdong Key medical subject.

\section{References}

1. Suzuki H: Laparoscopic adrenalectomy for adrenal gland carcinoma and metastases. Curr Opin Urol 16: 47-53, 2006.

2. Yoshitomi S and Tsuji H: A case of recurrent breast cancer with solitary adrenal gland metastasis treated with surgery and endocrine therapy. Gan To Kagaku Ryoho 39: 2074-2076, 2012 (In Japanese).
3. Do YR, Song HS and Kim IH: Adrenalectomy for metastatic disease to the adrenal gland from gastric cancer: Report of a case. Korean J Intern Med 22: 18-20, 2007.

4. Toikkanen S, Pylkkänen L and Joensuu H: Invasive lobular carcinoma of the breast has better short- and long-term survival than invasive ductal carcinoma. Br J Cancer 76: 1234-1240, 1997.

5. Liu XJ, Shen P, Wang XF, Sun K and Sun FF: Solitary adrenal gland metastasis from invasive ductal breast cancer: An uncommon finding. World J Surg Oncol 8: 7, 2010.

6. Eren OO, Ordu C, Selcuk NA, Akosman C, Ozturk MA, Ozkan F, Gokce O and Oyan B: Bilateral synchronous adrenal gland metastasis of invasive ductal carcinoma treated with multimodality therapy including adrenalectomy and oophorectomy. J Oncol Pharm Pract: pii: 1078155214551314, 2014.

7. Shen WT, Sturgeon C and Duh QY: From incidentaloma to adrenocortical carcinoma: The surgical management of adrenal gland tumors. J Surg Oncol 89: 186-192, 2005.

8. Akhtar K, Sherwani R and Kahkhashan E: Carcinoma breast metastasis to the suprarenal gland: An unusual presentation. Pol J Pathol 63: 284-285, 2012.

9. Andjelić-Dekić N, Božović-Spasojević I, Milošević S, Matijašević M and Karadžić K: A rare case of isolated adrenal gland metastasis of invasive ductal breast carcinoma. Srp Arh Celok Lek 142: 597-601, 2014.

10. Borst MJ and Ingold JA: Metastatic patterns of invasive lobular versus invasive ductal carcinoma of the breast. Surgery 114: 637-641; discussion 641-642, 1993.

11. Zografos GN, Perysinakis I, Kyrodimou E, Kassi E and Kaltsas G: Surgical treatment of potentially primary malignant adrenal gland tumors: An unresolved issue. Hormones (Athens) 14: 47-58, 2015.

12. Kajáry K, Molnár P, Lengyel Z and Szakáll S Jr: Port-site metastasis after laparoscopic removal of an isolated adrenal gland metastasis in a patient with breast cancer detected with FDG PET/CT. Clin Nucl Med 39: 203-205, 2014.

13. Shoji S, Usui Y, Nakano M, Hanai K, Sato H, Uchida T and Terachi T: Surgical management of metastatic adrenal gland tumors: Decision-making factors in imaging. Oncol Lett 1: 967-971, 2010.

14. Öztürk H: Bilateral synchronous adrenal gland metastases of renal cell carcinoma: A case report and review of the literature. Oncol Lett 9: 1897-1901, 2015. 